\title{
Endoscopic trans-sphenoidal drainage of pituitary abscess: a rare case report
}

\begin{abstract}
Background: Pituitary abscess is less common but life threating which is usually misdiagnosed as pituitary tumor pre-operatively, in spite of radiological advancement. Definite diagnosis is possible only postoperatively.
\end{abstract}

Material and methods: we report a case of 40years old woman, admitted in our department with symptoms of headache, visual impairment and pituitary dysfunction. MRI brain revealed sellar and supra-sellar mass consistent with pituitary tumor. She eventually underwent endoscopic trans-sphenoidal removal. Per operatively pus was evident. A month later, she was well and fully recovered pituitary function.

Results: The histopathological examination confirmed presence wall of abscess cavity, however, Culture showed no growth.

Conclusion: This case report gives emphasis on the importance of considering pituitary abscess as a differential diagnosis in patients with sella/supra-sellar mass. Early diagnosis and surgical management of a pituitary abscess significantly decreases morbidity and mortality, and it also helps to establish the definite diagnosis.
Volume 4 Issue 3 - 2017

\author{
Haradhan Deb Nath,' Rajeev Shah,' \\ Sudip Barua, ${ }^{2}$ KM Tarikul Islam,' Mahesh \\ Chaudhary, ${ }^{3}$ Mohammad Shahnawaz Bari,' \\ Kanak Kanti Barua' \\ 'Department of Department of Neurosurgery, Bangabandhu \\ Sheikh Mujib Medical University, Bangladesh \\ ${ }^{2}$ Department of Neurosurgery, National Institute of \\ Neuroscience, Bangladesh \\ ${ }^{3}$ Department of Radiology \& Imaging, Bangabandhu Sheikh Mujib \\ Medical University, Bangladesh
}

Correspondence: Haradhan Deb Nath, Department of Neurosurgery, Bangabandhu Sheikh Mujib Medical University, Bangladesh,Email dr.haradhan@yahoo.com

Received: September 22, 2017| Published: October 09, 2017

Keywords: pituitary abscess, pituitary adenoma, endoscopy, trans-sphenoidal

Abbreviations: DI, diabetes insipidus; TSS, trans-sphenoidal excision; ESR, erythrocyte sedimentation rate; CRP, c- reactive protein; $\mathrm{ACTH}$, adrenocorticotropic hormone; MRI, magnetic resonance imaging; T1WI, T1 weighted image; T2WI, T2 weighted image; CT, computed tomography

\section{Introduction}

Pituitary abscess is a rare, first case reported by Simmonds in 1914, accounts for less than $1 \%$ in seller region lesion. ${ }^{1,2}$ The most common symptom is nonspecific pattern of headache $(70-92 \%)$, followed by anterior pituitary dysfunction, central diabetes insipidus (DI), visual impairment, fever, meningeal irritation and nonspecific symptoms like vertigo. ${ }^{3-5}$

It may occur either de novo or as a result of hematogenous spread or spread from a contiguous focus of infection such as meningitis and sphenoiditis. ${ }^{3}$ The most commonly isolated pathogens are Staphylococcus spp. and Streptococcus spp., followed by Neisseria spp., Micrococcus, Citrobacter spp., Escherichia coli, Brucella, Salmonella, Corynebacterium \& Mycobacterium. ${ }^{5}$ However, in immunosuppressed patients, Aspergillus, Candida and Histoplasma are the most frequent pathogens. ${ }^{6}$ Pre-operative diagnosis is difficult because of rarity of disease, nonspecific symptoms and ring enhancing other pituitary lesions. ${ }^{7}$ Endoscopic Trans-sphenoidal excision (TSS) of the lesion with decompression of the sella is the most effective and safe approach for patients presenting with mass effect, followed by antibiotics for 4-6weeks, seems most acceptable treatment modalities in most of the literatures. ${ }^{8-10}$

\section{Case report}

A 40years old female presented in Department of Neurosurgery with complains of mild headache occasionally for 4 months, which was generally relieved by taking paracetamol. She had blurring of vision, generalized weakness and amenorrhea for 3 months. She had no significant past history. She was non-smoker and had no history of alcohol consumption. She had menarche at the age of 14years. She was married and had healthy children. On her examination, she was obese (with Basal Metabolic Index=-32.3), vital signs were within normal limit. All systemic examinations were normal including neurological examination except cranial nerve II i.e. bitemporal hemianopia. The patient had mild hirsutism over the face and the abdomen. There was no galactorrhea, and secondary sexual characteristics were unremarkable.

Laboratory investigation including Complete Blood Count, biochemical profile and inflammatory markers, including erythrocyte sedimentation rate (ESR) and C- reactive protein (CRP) levels including urine specific gravity and chest $\mathrm{x}$-ray were all within the normal range. Hormonal evaluation revealed mildly elevated serum prolactin levels of $31.16 \mathrm{ng} / \mathrm{mL}(1.39-24.2 \mathrm{ng} / \mathrm{ml})$, decreased serum thyroid-stimulating hormone (TSH) $0.05 \mu \mathrm{IU} / \mathrm{mL}$ (normal: $0.47-5.01$ ) with decrease free thyroxine (fT4) $5.35 \mathrm{pmol} / \mathrm{L}$ (normal: 9.14-23.81), fT3-1.32(2.62-5.7pmol/L and low morning serum cortisol of $2.6 \mathrm{nmol} / \mathrm{L}$ (normal:101.2-535.7nmol/L) with normal adrenocorticotropic hormone (ACTH) $13.9 \mathrm{pg} / \mathrm{ml}$ (normal: up to $46 \mathrm{pg} / \mathrm{mL}$ ).

Visual evaluation with Humphrey's perimeter revealed bitemporal hemianopia. Her Magnetic Resonance Imaging (MRI) of brain showed a mass measuring about $24 \mathrm{mmX} 22 \mathrm{~mm}$ in sellar/supra-sellar which was slightly hypo-intense lesion in T1 weighted image (T1WI), hyper-intense in T2 weighted image (T2WI) and after gadolinium contrast administration mild ring enhancement of lesion was seen. The mass was compressing the internal carotid arteries and optic chiasma (Figure 1). Hence, the patient was diagnosed possibly as Pituitary macroadenoma. 


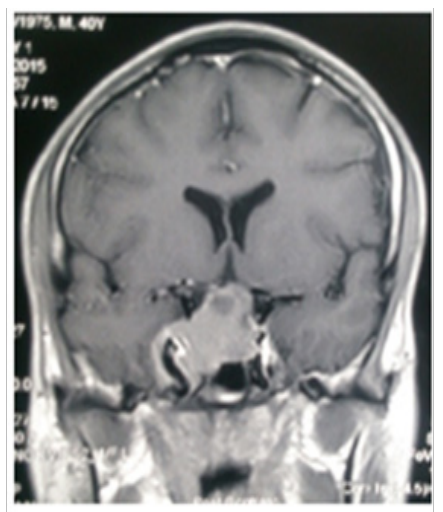

Figure I MRI of brain contrast/coronal, mild ring enhancement of lesion seen in sellar/supra-sellar compressing the internal carotid arteries and optic chiasma.

\section{Pre-operative period}

Her hormonal deficiency i.e. thyroid hormone and cortisol were corrected with thyroxin and hydro cortisone respectively. Operation and post-operative period: She underwent through endoscopic transsphenoidal removal of tumor. Per-operatively significant amount of yellowish colored pus was found with fibrotic tissue (Figure 2) and capsule, which was drained and sent for histopathological examination and culture. Postoperatively, she was managed with empirical intravenous antibiotics Ceftriazone and Amaikacin which were continued for 6 week and followed by 4weeks' oral antibiotic. No growth was found in culture and histopathology report was consistent with presence of wall of lesion. Hormone replacement was continued. She developed transient diabetic mellitus in early post-operative which was managed with Inj. Vasopression. Her immediate contrast enhanced Computed Tomography (CT) scan showed no evidence of existence of pre-operative lesion (Figure 3).

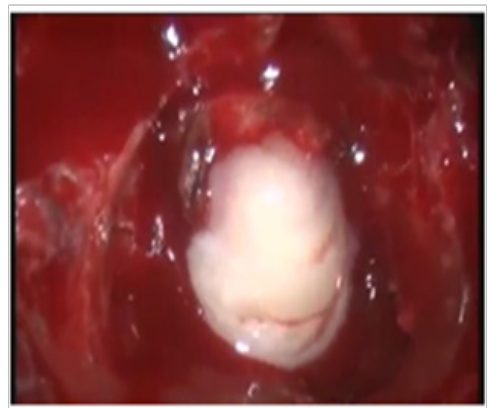

Figure 2 Endoscopic intra-operative picture shows yellowish white colored pus.

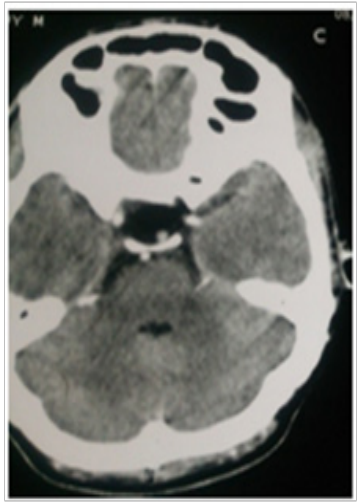

Figure 3 Post-operative CT scan of brain axial contrast, normal sellar and supra-sellar region.

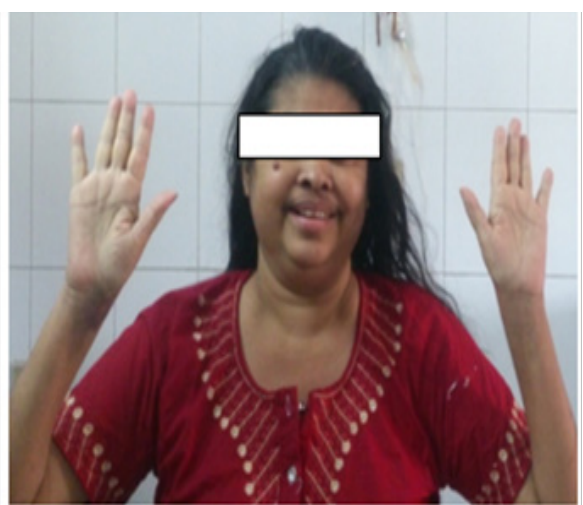

Figure 4 Postoperative photograph of patient.

\section{Outcome and follow up}

One month after surgery, while on cortisol and thyroxin (replacement therapy), her morning cortisol and TSH, T3, T4 were within normal range. So, hormonal replacement therapy was discontinued. Visual field examination was normal and there was no bitemporal hemianopia, and had fully recovered and regular menstrual cycles resumed after 2 months.

\section{Discussion}

A 40years female without any signs of infection and fever with radiological diagnosis of pituitary adenoma came up with the final diagnosis of pituitary abscess per intraoperative. Pituitary abscess accounts for less than $1 \%$ of all pituitary disease. Primary pituitary abscess occurs due to hematogenous seeding or by direct extension of adjacent infection, either in the CSF or in the sphenoid sinus, and more rarely as a complication of thrombosis of the cavernous sinus. ${ }^{11}$ Secondary pituitary abscesses occur in a pre-existing lesion, such as an adenoma, a craniopharyngioma or a Rathke cleft cyst. Other risk factors are underlying immunocompromised condition, previous pituitary surgery or irradiation of the pituitary gland. ${ }^{1}$

In this case, it was seen de novo like Jain et al where 5 cases out of 6 had no history of fever and sign of infection. No growth was found in culture despite no preoperative administration of antibiotics. Most common organism are Staphylococcus spp. and Streptococcus spp., followed by Neisseria spp., Micrococcus, Citrobacter spp., Escherichia coli, Brucella, Salmonella, Corynebacterium and Mycobacterium. However, in immunosuppressed patients, Aspergillus, Candida and Histoplasma are the most frequent pathogens. ${ }^{5,6}$

This case presented nonspecific pattern of headache, anterior pituitary dysfunction, visual disorders which accounts for 70$92 \%, 54-85 \%, 27-50 \%$ respectively and other symptoms may occur like central diabetic insipidus, fever with meningeal irritation and systemin illness with vertigo..$^{3-5}$ Commonly CT scan enlargement of the sella turcica and a well-defined low-attenuation rounded lesion demonstrating ring-enhancement ${ }^{4}$ and MRI iso or hypo-intense lesion on T1WI, iso or hyper-intense on T2WI and ring enhancement on gadolinium administration ${ }^{4,12}$ but in this case mild ring enhancement which signifies lack of matured capsule. Differential diagnosis of sellar cystic lesions include pituitary adenoma, carcinoma, abscess, arachnoid cyst, colloid cyst, Rathke cleft cyst, craniopharyngioma and metastasis. ${ }^{13}$

Trans-sphenoidal excision (TSS) of the lesion with decompression of the sella is the most effective and safe approach for patients presenting with mass effect. ${ }^{8-10}$ Endoscopic trans-sphenoidal is most 
favored over craninal because of more invasive and has a greater complication rate including bleeding, CSF infection, and thus longer hospitalization. ${ }^{14}$ Empirical treatment with antibiotics is indicated while awaiting microbiology and histological confirmation. Hence, it is important to do a Gram-staining and culture of the pus and to treat with appropriate antibiotics in the postoperative period to reduce the risk of recurrence. Hormone replacement therapy is administrated based on hormone deficits of the pituitary gland. ${ }^{14,15}$ We have treated out patient with empirical antibiotics.

Early diagnosis and treatment decreases mortality from $45 \%$ to $10 \% .{ }^{3,16}$ Recovery of vision and endocrine function were seen in a month in this case. However recovery period of the patient depends upon duration of symptoms.

\section{Conclusion}

Pituitary abscess is a rare disease. Pre-operative diagnosis is difficult because of rarity of disease, nonspecific symptoms and ring enhancing another pituitary lesions. One of the differential diagnosis of cystic supra-sellar mass is pituitary abscess. Early diagnosis and treatment decreases morbidities and mortalities significantly. Endoscopic trans-sphenoidal approach is the treatment of choice, followed by appropriate antibiotics for 4-6weeks.

\section{Acknowledgements}

None.

\section{Conflict of interest}

Author declares that there is no conflict of interest.

\section{References}

1. Jain C, Varma A, Mahapatra AK. Pituitary abscess: a series of six cases. British Journal of Neurosurgery. 1997;11(2):139-143.

2. Zur SM. Pathologe der Hypophysis. Verh Dtsch Ges Pathol. 1997;11:139-143.

3. Zhang X, Sun J, Shen M, et al. Diagnosis and minimally invasive surgery for the pituitary abscess: a review of twenty nine cases. Clin Neurol Neurosurg. 2012;114(7):957-961.
4. Liu F, Li G, Yao Y, et al. Diagnosis and management of pituitary abscess: experiences from 33 cases. Clin Endocrinol (Oxf). 2011;74(1):79-88.

5. Vates GE, Berger MS, Wilson CB. Diagnosis and management of pituitary abscess: a review of twenty-four cases. J Neurosurg. 2001;95(2):233-241.

6. Iplikcioglu AC, Bek S, Bikmaz K, et al. Aspergillus pituitary abscess. Acta Neurochir (Wien). 2004;146(5):521-524.

7. Walia R, Bhansali A, Dutta P, et al. An uncommon cause of recurrent pyogenic meningitis: pituitary abscess. BMJ Case Reports. 2010.

8. De Divitiis E, Cappabianca P, Cavallo LM. Endoscopic transsphenoidal approach: adaptability of the procedure to different sellar lesions. Neurosurgery. 2002;51(3):699-705.

9. Koutourousiou M, Kontogeorgos G, Seretis A. Non-adenomatous sellar lesions: experience of a single centreand review of the literature. Neurosurg Rev. 2010;33(4):465-476.

10. Carpinteri R, Patelli I, Casanueva FF, et al. Pituitary tumours: inflammatory and granulomatous expansive lesions of the pituitary. Best Pract Res Clin Endocrinol Metab. 2009;23(5):639-650.

11. Dutta P, Bhansali A, Singh P, et al. Pituitary abscess: Report of four cases and review of literature. Pituitary. 2006;9(3):267-273.

12. Danilowicz K, Sanz CF, Manavela M, et al. Pituitary abscess: a report of two cases. Pituitary. 2008;11(1):89-92.

13. Sabbah P, Bonardel G, Herve R. CT and MRI findings in primary pituitary abscess: a case report and review of literature. J Neuroradiol. 1999;26(3):196-199.

14. Ciappetta P, Calace A, D’Urso PI, et al. Endoscopic treatment of pituitary abscess: two case reports and literature review. Neurosurgical Review. 2008;31(2):237-246

15. Dalan R, Leow MK. Pituitary abscess: our experience with a case and a review of the literature. Pituitary. 2008;11(3):299-306.

16. Blackett PR, Bailey JD, Hoffman HJ. A pituitary abscess simulating an intrasellar tumor. Surg Neurol. 1980;14(2):129-131. 